\title{
Color Symmetry, Semigroups, Fractals ${ }^{\dagger}$
}

\author{
Vladimir R. Rosenfeld \\ Mathematical Chemistry Group, Department of Marine Sciences, Texas A\&M University at Galveston, \\ Galveston, TX 77553-1675, USA \\ Instituto de Ciencias Matematicas (ICMAT) CSIC \\ C/ Nicolás Cabrera, $n^{\circ}$ 13-15 Campus de Cantoblanco, UAM, 28049 Madrid, Spain \\ (E-mail: rosenfev@tamug.edu,vladimir_rosenfeld@yahoo.com) \\ RECEIVED MAY 25, 2013; ACCEPTED AUGUST 6, 2013

\begin{abstract}
We discuss within one common context topics of color symmetry, using semigroups for generalization of symmetry, fractals, and some naturally occurring interrelations thereof. This free essay is emotionally inspired by the impressive scientific work of Prof. Douglas Jay Klein and his numerous collaborators, who contributed into diverse fields of chemistry, physics, and mathematics. (doi: 10.5562/cca2303)

Keywords: color symmetry, automorphism (or symmetry) group, equivalence class, orbit, normal subgroup, normalizer, semidirect product of groups, transitive group, orbit stabilizer, endomorphism, semigroup, endomorphism monoid, strong endomorphism, fractal, semifractal
\end{abstract}

\section{INTRODUCTION}

We discuss herein in a free form three topics: color symmetry, the use semigroups for the generalization of symmetry, fractals, and some interrelations thereof. Such a set of subjects may be chosen due to practical reasons that come from chemistry and physics studying real objects and seeking theoretical explanation of their properties.

\section{COLOR SYMMETRY}

Let us start with one case of color symmetry (CS). ${ }^{1,2}$ It has the following feature. In a proper 'polychromatic' case, an object $\mathcal{O}$ in general possesses two types of symmetry. The operations of symmetry of the first type, if any, act quite normally just on parts of $\mathcal{O}$ which are colored with the same color. Say, a perpendicular plane passing through a diagonal of a chessboard, with white and brown cells, acts as a normal element of (mirror) symmetry which maps all white cells lying on either side of it into white ones on the other side and, similarly, does all brown cells (and halves of diagonal cells). However, the operations of the second type (or operations of color symmetry) necessarily permute (some) parts colored with distinct colors. Say, a perpendicular plane crossing the centers of opposite edges of a chessboard maps all white cells into brown ones, and vice versa. Such an operation, unforeseen by the usual automorphism, or symmetry, group $\hat{H}$ of $\mathcal{O}$, can be realized only if one colors up all cells with the same color. In the second case, there acts a bigger automorphism group $G=$ Aut $\mathcal{O}$ of $\mathcal{O}$ which "daltonistically" neglects all differences in colors and, instead of this, more adequately takes into account geometric features of $\mathcal{O}$ (or others, say, topological ones). In a nondegenerate chessboard's case, naturally, $\hat{H} \subset G$ but, in a wider context, we shall write $\hat{H} \subseteq G$. This general context is actual when, on the contrary, one takes a monochromatic object, say, a graph, and studies symmetries of its colorings - some colorings may obey the maximum symmetry of the original unchromatized object, especially if this object has a small symmetry group.

Out of many natural objects which may possess color symmetry, crystals are especially interesting. ${ }^{1,2}$ We want to avoid all situations where coloration of these objects is used in model considerations, such as the case of large unit cells (LUC) ${ }^{2}$ with a rather complex pattern of distribution of spin particles therein. For our discussion, quite normal instances are adequate. Note that a body-centered and face-centered cubic lattices (BCC and FCC) can be considered as constructions from two and four simple cubic lattices (SC), respectively. Apparently, these interpenetrated SC's play roles of sublattices in BCC and FCC; and in both pristine lattices, there are respective translations that transfer

\footnotetext{
$\dagger$ Dedicated to Professor Douglas Jay Klein on the occasion of his $70^{\text {th }}$ birthday.
} 
each of their sublattices into any other. At this point of exposition, there is no color symmetry at all. We just fill all such sublattices of a crystal, each, with a distinct sort of atoms, none of mentioned translations can act. Otherwise, the transformation of one sublattice into another might mean a permutation of sorts of atoms. Or, if to use colors in a lieu of atoms, we must say that in such a case, forbidden by chemistry, translations of a geometric lattice can perform operations of color symmetry. In general, we assume that such operations may permute only certain colored parts of $\mathcal{O}$ and fix the others. In any event, we come to an algebraic setting of our problem.

Described sublattices of a crystal are only one case of what are called equivalence classes, or orbits, of objects. ${ }^{3}$ Every automorphism, or symmetry, group $G$ (Ref. 3) acting on a nonempty countable set $X$ induces the distribution of elements of $X$ into orbits. Similarly acts every subgroup $H \subseteq G$, which in general induces different orbits (where "different" means also that the number of these is greater than in the former case of $G$ itself). Here, we must point out the following. Along with the mentioned subgroup $H$, there may, in general, exist other subgroups, say $H^{\prime}, H^{\prime \prime}, \ldots$ which induce the same orbits that $H$, on $X$. Therefore, usually, they find the biggest $\hat{H}$ of all these coorbital subgroups $(\hat{H} \supseteq H$, $\left.H^{\prime}, H^{\prime \prime}, \ldots\right)$ which does always exist and contains all the others, if any. In a special literature, such a subgroup $\hat{H}$ is called (after Rota and Smith) a closed subgroup or the closure of $H, H^{\prime}, H^{\prime \prime}, \ldots$ (Refs. 4 and 5) The existence of the notion of a closed subgroup plays a crucial role in our exposition below.

One may use different colors for orbits that $\hat{H}$ induces on $X$. If all elements of each orbit share one common color, then the overall coloring of all orbits conserves the original symmetry described by the subgroup $\hat{H}$, at least as a subsymmetry of the obtained coloring. Note that in case of $\hat{H}=G$ all colorings of intact orbits exactly possess the maximum symmetry described by $G$; but what is worth specially mentioning is that this maximum symmetry, even with the possibility to obtain colorings, leaves no room for color symmetry, if defined as above. The matter is that there remains no 'spare' element of symmetry that might transfer orbits of one colors into orbits of other ones - and even unchromatized orbits at all. For realization of color symmetry, as it was with permutations of sublattices with distinct atoms, one must necessarily have just a proper subgroup $\hat{H} \subset G$ (Ref. 3) and, of course, entirely colored orbits of it - and even this is not yet a suficient condition, in general.

Recall that $H_{1} \unlhd H_{2}$ denotes the "inclusion or equality" for a normal subgroup $H_{1} \subseteq H_{2}$ (Ref. 3). In what closed subgroups $\hat{H} \subseteq G$ are peculiar is the following property thereof: The normalizer $N_{G}(\hat{H})$ (Ref. 3) of a closed subgroup $\hat{H}\left(\hat{H} \unlhd N_{G}(\hat{H}) \subseteq G\right)$ is the maximum subgroup, in $G$, that permutes (or fixes) intact orbits of
$\hat{H}$ on $X$ (Ref. 5). (Accordingly, just orbits of the same cardinality can mutually exchange their places.) In general, coorbital subgroups $H, H^{\prime}, H^{\prime \prime}, \ldots$ of it, if any, do not have this property of their normalizers. In case of color symmetry, just elements $g \in\left(N_{G}(\hat{H}) \backslash \hat{H}\right)$ and only these permute intact colored orbits, including the abovementioned SC-sublattices of BCC and FCC lattices. Thus, an extended necessary condition for $N_{G}(\hat{H})$ to contain operations of color symmetry is $\hat{H} \triangleleft N_{G}(\hat{H}) \subseteq$ $G$, which is not suficient, either. The 'most impressive instance' (MII) is, certainly, wherein all orbits are colored with different colors and each of them can be permuted with any other from these orbits. The above SCsublattices of BCC and FCC lattices are exactly such.

As known, $G_{\mathrm{SC}}=T_{1} \lambda O_{h}$, where $\mathrm{G}_{\mathrm{SC}}$ is a full space-symmetry group of SC, $T_{1}\left(\left|T_{1}\right|=\infty\right)$ is a translation group of SC, $O_{h}\left(\left|O_{h}\right|=48\right)$ is the point-symmetry group of octahedron and cube, and " $\lambda$ " denotes that GSC is a semidirect product of $T_{1}$ and $O$, with $T_{1}$ being a normal subgroup of $G_{\mathrm{SC}}\left(T_{1} \triangleleft G_{\mathrm{SC}}\right)$. Moreover, $G_{\mathrm{BCC}}=$ $T_{2} \lambda O_{\mathrm{h}}$, where $T_{1} \triangleleft T_{2}$ is also a normal subgroup of the translation group $T_{2}$ of index $\left|T_{2}: T_{1}\right|=2$; and $G_{\mathrm{FCC}}=T_{4}$ $\lambda O_{h}$, where $T_{1} \triangleleft T_{4}\left(\left|T_{4}: T_{1}\right|=4\right)$. But rather interesting to us are the following relationships: $G_{\mathrm{SC}} \triangleleft G_{\mathrm{BCC}}$ and $G_{\mathrm{SC}} \triangleleft G_{\mathrm{FCC}}$, whence follow their equivalents $N_{\mathrm{BCC}}\left(G_{\mathrm{SC}}\right)$ $=G_{\mathrm{BCC}}$ and $N_{\mathrm{FCC}}\left(G_{\mathrm{SC}}\right)=G_{\mathrm{FCC}}$, respectively. See below.

Now, recall that a transitive permutation group $G$ (Ref. 3) induces only one orbit on $X$, which coincides with $X$. Using this general definition and the last two expressions above as a specific case, we formulate a necessary and suficient condition for the realization of what we called the 'most impressive instance' (MII) of color symmetry above. Namely, it reads as follows: $\hat{H}$ describes the MII of color symmetry iff (if and only if) $\hat{H} \triangleleft G$, where $G$ is a transitive permutation group acting on $\mathrm{X}$. Or in words, a closed subgroup $\hat{H}$ should be a proper normal subgroup ${ }^{3,5}$ of a transitive permutation group $G$. In particular, just this property of $\hat{H}=G_{\mathrm{SC}}$ was discussed by us above. Lastly, note that from $\hat{H} \triangleleft \mathrm{G}$ automatically follow conditions imposed on the cardinality $|X|$ of a finite set $X$, since $\hat{H}$ thus distributes $X$ into $s$ orbits of the same size $|\mathrm{X}| / s$, where $s$ obligatorily divides the index $|\mathrm{G}: \hat{H}|=|\mathrm{G}| /|\hat{H}|$. Even more, since $\hat{H}$ is a stabilizer ${ }^{3}$ of each $\hat{H}$-orbit in the set $\hat{H} \backslash \backslash X$ of all $\hat{H}$ orbits on $X$, s exactly equals $|\mathrm{G}: \hat{\mathrm{H}}|$ (Ref. 3). Therefore, as a consequence, $|\mathrm{X}|$ cannot be a prime number - only the product of at least two numbers - a circumstance that was not taken into account in general. Non-MII cases of color symmetry are rather complicated and demand special studies thereof, which may, however, be more interesting than the MII.

\section{SEMIGROUPS AND SYMMETRY}

First, recollect the usual symmetry in nature which (whether color or not) is described by symmetry groups; 
therefore, we shall term it 'group symmetry'. For all discrete sets $X$ of objects, such as vertices of polyhedra, atoms in a molecule or crystal, etc., a group of symmetry can be represented by a permutation group $G$. The operations of symmetry which are described by $G$ are one-one, i.e., are one-valued and reversible. Accordingly, every two different elements $x_{1}, x_{2} \in X$ are obligatorily transferred into two different elements $g x_{1}$ and $g x_{2}$, where $g \in G$ and $g x_{i}(i=1,2)$ denotes the image of $x_{i}$ under an automorphism (or symmetry operation) $g$. Thus, disregarding which different elements $x_{1}$ and $x_{2}$ are taken, $g x_{1}$ and $g x_{2}$ cannot be equal.

Now, instead allow our (one-valued) mapping $g:=$ $g X \mapsto X$ to send two (or more) elements into one common image. That is, we allow $x_{1} \neq x_{2}$ such that $g x_{1}=$ $g x_{2}$. Apparently, here, $g$ cannot be accompanied by a one-valued inverse mapping $g^{-1}$; the latter does not simply exist. The mapping $g$ with such a 'contracting' property is called an endomorphism..$^{6,75}$ Usually, automorphisms (which are all invertible) are considered as a specific case of endomorphisms; but a proper endomorphism is not an automorphism. The set of all endomorphisms of $X(|X|=n)$ comprises the symmetric semigroup $M_{n}$ (Refs. 6 and 7), which is a generalization of the symmetric group $S_{n}$, on $X$. Every semigroup $S$ is isomorphically represented by a subsemigroup of a symmetric semigroup $M_{n}(n \geq|S|){ }^{6,7}$

In a natural world, automorphisms and proper endomorphisms may have another difference. In particular, rotations of a solid as a whole are really performed; and this does not require to disintegrate the object during such actions. Operations involving internal rotations of nonrigid molecules (say, of hydrocarbons) or rotation-reflection axes of symmetry of solids already require to alter a mutual arrangement of parts of an object at least in imagination (or physically at intermediate stages). But all the same, such operations finally produce an intact copy of the object which is fully identical to its original. From this naturally follows that automorphisms conserve the quantity of matter in nature.

Contrary to automorphisms, proper endomorphisms always irreversibly destroy the original object and compress its content to a proper part thereof. In particular, any number of symmetryequivalent parts may be compressed into one of them; but, in general, unequal parts may be compressed, as well. An elementary example is an arbitrary object with mirror symmetry (a drawing, molecule, etc.). Say, superimposition of one half of a molecule $\mathrm{H}_{2} \mathrm{O}$ on the other results in one "doubly-dense" half HO.

It is clear that such symmetry operations on material objects may exist just in our imagination - but they unrestrictedly may be applied to any abstract objects (models of natural objects). In chemical practice, two atoms cannot be contracted into one atom; but when we begin to consider a respective molecular graph, two vertices representing these atoms may well be glued into one vertex, for the sake of mathematical reasoning and manipulations. Just a rigorous physical law of the conservation of matter prohibits proper endomorphisms to occur as annihilators in material nature. That is why experimentalists could not observe proper endomorphisms in their material investigations, in contrast to automorphisms (say, involving rotational axes). Nonetheless, endomorphisms abound in nature in a wide range of regularities and constructional patterns thereof. The search for any regularities in nature is often engendered on analysis and imaginary disintegration of its objects.

Add also that groups of automorphisms describe all invariant transformations and steady equilibria, while in semigroups the proper endomorphisms describe possible irreversible directions of a process. Thereby, an experimentalist studying such phenomena of a physical world may have this as a hint for choosing suitable mathematical theories that might help in the rigorous exposition of results of investigations. In particular, 'black holes' have quite an endomorphic, irreversible feature - to attract all objects and not give them back. Why not come to them sometime with a semigrouptheoretical lever?

As well as in the case of groups, ${ }^{8}$ all semigroups can isomorphically be represented as endomorphism semigroups of pertinent algebraic objects, e.g., graphs. ${ }^{9}$ Without delving into details, note that there are known several possible types of endomorphisms of graphs. But what all these types share in common is the condition that each endomorphism $\varepsilon$, from a semigroup $S=\operatorname{End} \Gamma$ of endomorphisms of a graph $\Gamma(\mathrm{V}, \mathrm{E})$, must conserve the adjacency of vertices. That is, $u \sim v(u v \in E)$ implies $\varepsilon u \sim \varepsilon v$. For a strong endomorphism $\sigma^{10-12}$ such an implication is reversible for both adjacency and nonadjacency: $u \sim v$ iff (if and only if) $\sigma u \sim \sigma v$ and $u \nsim v$ iff $\sigma u \nsim \sigma v$, but not for other types of endomorphisms. Recall that a monoid ${ }^{6,7}$ is semigroup $S^{1}$ with a unit (which may be added to a semigroup $S$ without it: $S^{1}=$ $S \cup 1$ ). If $M$ is a monoid of endomorphisms of a sequence of symbols with some regular subsequence (hidden among other symbols), the closed-submonoidnormalizer scheme may develop this subsequence (figuratively, like one developed a photographic film in the near past). That is, a semigroup-theoretical approach is, in particular, needed for finding hidden regularities with symmetries. i.e., in a physical pattern recognition, by which we do not restrict to the use of vectors of descriptors, but of full patterns of physical objects. Now, we turn to the next topic which is intimately related to what we considered above. 


\section{FRACTALS}

In nature or mathematician's imagination, fractals ${ }^{13-20}$ are objects that stay selfsimilar under scaling both up and down, though the very term is due to the fractional geometric dimensionality of this objects, rather than their scale. The definition here includes also a condition that such scaling in either direction needs must do unrestrictedly; thus, every such fractal is described by an infinite group. To a chemist, it is evident that scaling of a molecular fractal (let us assume that this may exist) is possible only down to atomic sizes, whereas it seems to be unbound while scaling up. Somehow or other, there exist objects whose scaling in either or even both directions is restricted, termed semifractals; for describing these, one needs to use a more general theory of semigroups.

The best commonly applicable representation of (semi)fractals is based on the composition of functions. Here, an elementary example follows. Take an arbitrary function $f(x)$ and iterate an infinite series $\mathcal{F}:=x, f(x)$, $f[f(x)], f\{f[f(x)]\}, \ldots=f_{0}, f_{1}, f_{2}, f_{3}, \ldots$. The application of the operator $f()$ consecutively to all members of $\mathcal{F}$ is tantamount to the shift by one position, in it, to the right. That is, $\mathcal{F}$ is a semifractal with an infinite monogenic (cyclic) semigroup $S$ of such shifts. In case of an invertible function, there also exists $f^{-1}(x)=\varphi(x)$; and one may extend the series $\mathcal{F}$ also in the other direction, consecutively considering on the left side of $x$ members $\varphi(x), \varphi[\varphi(x)], \varphi\{\varphi[\varphi(x)]\}, \ldots$. It produces a fractal $\mathcal{F}^{*}$ with a group $G$ of left and right shifts due to iterating $\varphi()$ and $f()$, respectively. Plenty of generalizations can be obtained using functions in many variables, considering the argument $x \bmod n \in \mathbb{N}$, etc. All of them are a priori taken into account by theory of semigroups (say, as semigroups of symmetry of such objects). Here, there is a wide perspective for applications of the latter.

As a case of $f(x)$ above, one may take an arbitrary $F$-polynomial $f(\Gamma ; x)$ of a graph $\Gamma$ (Refs. 21 and 22$)$ and formally produce the entire series $\mathcal{F}$ or, maybe, even $\mathcal{F}^{*}$. Here, a very interesting practical question arises: Whether there exist such graphs $\Gamma_{i}(i \geq 1)$ for which their polynomials $f\left(\Gamma_{i} ; x\right)$ coincide with respective iterated polynomials $f_{i}(\Gamma ; x)$ in $\mathcal{F}$ ? For each specific $F$ polynomial, this question asks about the possibility to find, if any, a respective operation $\odot$ acting on $\Gamma_{i}$ (maybe, together with some auxiliary graphs) and producing the next homolog $\Gamma_{i+1}\left(i \geq 0 ; \Gamma_{0}:=K_{1} ; \Gamma_{1}:=\right.$ $\Gamma)$. To the best of our knowledge, such instances involving the $F$-polynomials have not (yet) been found but found for the reduced independence polynomial. ${ }^{23}$ That is, an idea of the simplest one-parameter semifractal of this type is realized in graph theory, which was yet earlier realized by Klein \& Seitz; ${ }^{24,25}$ Seitz, Klein, \& Hite; ${ }^{26}$ and Klein, Živković, \& Balaban ${ }^{27}$ in physical chemistry. However, it is more easily realized if one refuses from consideration of polynomials and only considers any recurrent procedure for constructing a graph series. Polynomials are easily treatable if to consider a two-parameter fractal, with substitution of two (different) functions at once - for two variables $x$ and $y$, rather than one $x$. A case of such fractals is a dendrimer graph series. ${ }^{28}$ However, we turn here to applications of semigroups to describing symmetry of certain crystals.

Usually, the construction of crystals is attributed to the presence of congruent unit cells having a parallelepipedal form. An instance of parallelepiped is a cube. A cube can be distributed into a set of smaller cubes of different sizes. Copying all proportions of sizes and a mode of packing of the original cube, one can distribute exactly in the same geometric fashion each of smaller cubes and, in principle, continue this process a finite or, conditionally, infinite number of times. One can construct in this way a Russian-doll, or matrëška, fractal. In general, the same procedure can be applied to a parallelepiped using the distribution into smaller parallelepipeds with the same ratios of edge lengths or using more general operations (including also nonproportional distributions). In any case, at any level of fractal cellularization, one may claim that every two corresponding atoms of a crystal lying on opposite faces of every cell should be the same, i.e., by full analogy with atoms of opposite faces of normal cells. Atoms inside cells are not so directly considered but may sooner or later fall onto certain faces; even if it does not happen, we may confine ourselves with considering only atoms lying on cells' faces.

Now, without any loss of generality, choose as an elementary case a SC lattice. Construct a symmetrical digraph from it, substituting a pair of opposite arcs for every segment joining two adjacent lattice knots and attaching an oriented selfloop to every knot. We may also color red, green, and yellow all parallel and antiparallel arcs pointed consistently with axes of abscissas, ordinates, and applicates, consecutively. Additionally, we may attach to every knot its three-dimensional coordinates $(x, y, z)$ and, similarly, specify types of elementary translations $( \pm 1,0,0),(0, \pm 1,0),(0,0, \pm 1)$ on each arc. So, we obtained a marked graph of the infinite translation group $T_{1}$ of SC. Usually, a literal notation of all knots and translations is employed; because of it, one may also say about an (algebraic) weighting of knots, arcs, and selfloops.

Now, consider a general case of an algebraically weighted graph of an arbitrary classical crystal lattice. For every consistently oriented walk from one knot to any other one or back to itself, define the weight of this walk as a product of weights of all arcs and selfloops which it passes, taking into account the number of times each arc and selfloop is passed. Then, if all weights are 
elements of a translation group, a total weight of a walk equals 1 iff this walk is a closed one (i.e., returning to a point from which it went out $)^{29,30}$ or if a walk corresponds to an integral linear combination of elementary translations.

Apparently, in case of a graph of a crystal lattice cellularized in a Russian-doll fractal fashion, a translation-group weighting renders unworkable. What may work instead is similarly employing of elements of a pertinent semigroup (monoid). In thus doing, the condition of the product of weights to be equal to the identity 1 of a translation group gradually generalizes to that of being equal to any idempotent $e\left(e^{2}=e e=e\right.$ ) (Refs. 6 and 7) of a respective monoid $M$ (semigroup) of symmetry and, then, even to an arbitrary element $g$ of a proper subsemigroup $S \subset M$ which has a strictly including it normalizer $N_{M}(S) \triangleright S$. As a result, all operations $h$ of $N_{M}(S)$ that do not belong to $S\left(h \in\left(N_{M}(S) \backslash S\right)\right)$ will permute (and, possibly, fix other $)^{5}$ sublattices of our matrëška-fractal crystal. These sublattices may be of distinct cardinalities (see 3 lines above Corollary 14.1 in Ref. 5) and quite bizarre in appearance - but they can predictably exist!

Add that the same semigroup-theoretical symmetry properties may also be encountered among noncrystal graphs. As to generalizing crystals, interesting generalizations may be cellular constructions of living beings and the very genomic sequences. ${ }^{31-33}$ In a more general context. ${ }^{34}$

We did not give any rigorous proof in our philosophical discussion but have touched upon a number of interesting questions that might each become a subject of a special rigorous research.

Acknowledgements. We are grateful to Prof. Stuart Whittington (Toronto, Canada) for carefully reading the manuscript and his linguistic suggestions. The support of the Welch Foundation of Houston, Texas (via grant BD-0894) is acknowledged.

\section{REFERENCES}

1. A. V. Shubnikov, N. V. Belov, and W. T. E. Holser, Color Symmetry, Pergamon Press, Oxford, 1964.

2. A. V. Shubnikov and V. A. Koptsik, Symmetry in Science and Art, New York, Plenum Press, 1974.

3. A. Kerber, Applied Finite Group Actions, Springer, Berlin, 1999.

4. G.-C. Rota and D. A. Smith, Ann. Sci. Norm. Super. Pisa. Cl. Sci. 4 (1977) 637-646.

5. V. R. Rosenfeld, MATCH Commun. Math. Comput. Chem. 43 (2001) 111-130 (see Erratum on p. 125: A lost multiplier s just after the sign $\Sigma$ in (16)!).
6. A. H. Clifford G. B. and Preston The Algebraic Theory of Semigroups, 2 vols, $2^{\text {nd }}$ ed., American Mathematical Society, Providence, R. I., 1967.

7. L. N. Shevrin, Semigroups, in: General Algebra, L. A. Skornyakov (Ed.), v.2, Nauka, Moscow, 1991, pp. 11-191. (In Russian.)

8. R. Frucht, Canad. J. Math. 1 (1949) 365-378.

9. Z. Herdlin and A. Pultr, Monatsch. Math. 69 (1965) 318-322.

10. U. Knauer and M. Nieporte, Archiv der Mathematik 52(6) (1989) 607-614.

11. V. R. Rosenfeld, MATCH Commun. Math. Comput. Chem. 20 (1999) 203-214.

12. V. R. Rosenfeld, Toward molecules with nonstandard symmetry, in: Diamond and Related Nanostructures, Mircea V. Diudea and Csaba L. Nagy (Eds.), Springer, Berlin, 2013, Ch. 14, pp. 275-285.

13. B. B. Mandelbrot, The Fractal Geometry of Nature, W. H. Freeman and Co., New York, 1982.

14. H.-O. Peitgen and D. Saupe (Eds.), The Science of Fractal Images, Springer-Verlag, New York, 1988.

15. H. Lauwerier, Fractals: Endlessly Repeated Geometrical Figures, Translated by Sophia Gill-Hoffstadt, Princeton University Press, Princeton NJ, 1991.

16. H. Jürgens, H.-O. Peitgen, and D. Saupe, Chaos and Fractals: New Frontiers of Science, New Springer-Verlag, New York, 1992.

17. M. F. Barnsley and H. Rising, Fractals Everywhere, Academic Press Professional, Boston, 1993.

18. J.-F. Gouyet, Physics and Fractal Structures (Foreword by B. Mandelbrot) Masson, 1996; and Springer-Verlag, New York, 1996.

19. K. Falconer, Techniques in Fractal Geometry, John Wiley and Sons, 1997.

20. C. A. Pickover (Ed.), Chaos and Fractals: A Computer Graphical Journey - A 10 Year Compilation of Advanced Research, Elsevier, 1998.

21. E. J. Farrell, J. Combin. Theory B 26(1) (1979) 111-122.

22. E. J. Farrell and V. R. Rosenfeld, J. of Math. Sci. (India) 11(1) (2000) 35-47.

23. J. I. Brown, C. A. Hickman, and R. J. Nowakowski, J. Combin. Theory B 87 (2003) 209-230.

24. D. J. Klein and W. A. Seitz, Proc. Natl. Acad. Sci. USA 80 (1983) 3125-3128.

25. D. J. Klein and W. A. Seitz, J. Physique Lett. 45 (1984) L-241L-247.

26. W. A. Seitz, D. J. Klein, and G. E. Hite, Discrete Appl. Math. 19 (1988) 339-348

27. D. J. Klein, T. P. Živković, and A. T. Balaban, MATCH Commun. Math. Comput. Chem. 29 (1993) 107-130.

28. V. R. Rosenfeld and M. V. Diudea, Internet Electron. J. Mol. Design 1(3) (2002) 142-156.

29. D. J. Klein and V. R. Rosenfeld, J. Math. Chem. 49(7) (2011) 1238-1244.

30. D. J. Klein and V. R. Rosenfeld, J. Math. Chem. 49(7) (2011) 1245-1255.

31. V. R. Rosenfeld, MATCH Commun. Math. Comput. Chem. 56(2) (2006) 281-290.

32. V. R. Rosenfeld, J. Theor. Chem. 1(1) (2013) submitted.

33. V. R. Rosenfeld, to be submitted.

34. V. R. Rosenfeld, Sci. Isr. Technol. Adv. 9(1) (2007) 56-65. 